\title{
Enhancement of Refractive Index Sensitivity in Photonic Crystal Waveguide-Based Sensors by Selective Infiltration
}

\author{
F. BAGCI AND B. AKAOGLU* \\ Ankara University, Faculty of Engineering, Department of Engineering Physics \\ 06100 Besevler, Ankara, Turkey
}

(Received November 2, 2012; in final form April 10, 2013)

\begin{abstract}
We present a liquid refractive index sensor based on a photonic crystal waveguide slab structure. Sensing mechanism employed in this study is based on the shift in cut-off wavelength as the lattice holes are selectively infiltrated. Three-dimensional plane-wave expansion and finite-difference time-domain methods are used to determine the band structure and transmission spectra, respectively. First, the sensitivity of the device is analyzed for the structure where only the first rows of holes adjacent to the line-defect are infiltrated. In addition, this analysis is repeated for a range of hole diameters. Second, the effects of infiltrated holes which are placed in the line-defect are investigated. As these infiltrated central holes are introduced, the proposed device exhibits 5.3 times improved sensitivity.
\end{abstract}

DOI: $10.12693 /$ APhysPolA.124.50

PACS: 42.70.Qs, 78.67.Pt, 07.07.Df, 42.79.-e

\section{Introduction}

Optical sensors have gained a lot of attention over time due to their high sensitivity to detect small changes in temperature, humidity, pressure, chemical composition, etc. Besides, optical sensors eliminate the need for fluorescent or radioactive labeling of molecules, which are relatively time-consuming, expensive and large area demanding [1]. Various label-free optical sensing techniques are proposed which are based on surface plasmon resonance [2], interferometry [3-5], waveguides [6, 7], optical fibers [8] and ring resonators [9-11]. Although, some of these techniques are now mature and even commercialized, these techniques are not very practical for on-chip integration with incorporation of light sources and detectors. In contrast, photonic crystals offer smaller sizes and mass fabrication of on-chip integrated circuits.

Photonic crystals (PC) are dielectric or metallic materials where a periodic modulation of the refractive index gives rise to a range of frequencies with prohibited transmittance in the PC. By changing the radius of central holes or omitting them completely, localized states can be created in the photonic band gap and a photonic crystal waveguide (PCW) can be formed. The guided mode propagates in the PC laterally by the intrinsic band gap effect and vertically by total internal reflection.

Recently a large number of groups have employed photonic crystal refractive index sensors in various architectures [12-23]. In general, the architecture of PC-based refractive index (RI) sensors can be categorized in three

*corresponding author; e-mail: akaoglu@eng.ankara.edu.tr groups as bulk PCs, PCWs and PC cavities. While the PC cavity sensors can have enhanced sensitivity and smaller sensing area, mass fabrication of the PC cavity sensors remain to be a challenge since any variation in the defect size can alter the resonance frequency and affect the sensitivity. In this respect, $\mathrm{PCW}$ designs remain to be much more robust under fabrication tolerances and can more easily be fabricated.

Detection of liquid or molecules in the PCW sensors can be performed by surface-based sensing or bulk index sensing [13]. In addition, rather than immersing the PC slab in a low-index fluid (bulk-index sensing), the infiltration of selected holes, which interacts more with the field, can be employed. The cut-off wavelength of a PCW is observed to be very sensitive to the changes in the surrounding environment [18]. Such a structure can be used as a sensor, whose principle of operation is based on the cut-off wavelength shift caused by the infiltration of the holes. Infiltration can be performed by various selective infiltration techniques, which have been experimentally demonstrated, such as using an integrated microfluidic circuit [24], an actuated microtip [25] or micropipette [26] with a high precision and good reproducibility.

In this study, water, ethanol, isopropanol and xylene are selected for analysis of the cut-off wavelength shift with respect to the cut-off wavelength for air. In some studies, the behavior of the cut-off or resonance wavelength shift under RI change is not evaluated [18, 20, 21]. However, to gain maximum performance from the sensor device, this characteristic should be revealed. In this study, a linear relation in cut-off wavelength versus ambient RI change is obtained over a RI range of 0.5.

In an earlier study, isopropanol is distinguished from xylene by a PC branched waveguide microfluidic detector where the input waveguide is a W1 line-defect waveguide 
while the two branches consist of waveguides with holes smaller than those in the bulk PC [22]. Buswell et al. enhanced the bulk index sensitivity $40 \%$ (from $88 \mathrm{~nm} / \mathrm{RIU}$ to $120 \mathrm{~nm} / \mathrm{RIU}$ ) by placing small size holes in the line-defect [20]. In this study, a much higher improvement of sensitivity ( 5.3 times) is achieved by selectively infiltrating only the holes in the line-defect (central holes). To the best of our knowledge, such a huge increase of sensitivity by inclusion and infiltration of central holes has not been reported.

\section{Design and modeling}

The investigated PC structures consist of circular air holes in a triangular lattice symmetry with a hole diameter $(d)$ of $0.76 a$ where $a$ is lattice constant. The $\mathrm{W} 1$ waveguide is formed by removing a row of holes in the $\Gamma-K$ direction. Recently, it has been shown that air-suspended membrane-type PCs have a greater sensitivity than PC slabs on a substrate due to the higher refractive index contrast [21]. Therefore, the Si PC slab $(n=3.47)$ in our study is suspended in air. Air-bridge photonic crystal structures can be practically fabricated by using selective wet etching techniques $[27,28]$ and can be easily integrated with microfluidics [29]. The height of the PC slab is selected as $h=0.6 a$ and seven rows of holes are introduced on each side of the line-defect. This design enables a large photonic band gap for TE-polarized light where the cut-off wavelength of the fundamental guided mode can be chosen around $1.5 \mu \mathrm{m}$. The lattice holes are selected to have a $0.76 a$ diameter value because it corresponds to larger photonic band gap as compared to smaller diameter values [30].

In the simulations, the polarization is assumed to be TE-even which has magnetic field component parallel to air hole axis. In the determination of band structure characteristics and mode field distributions, MIT's photonic band package (MPB), which uses plane-wave expansion method with periodic boundary conditions, is adopted [31]. The computations are carried out on a $a / 16 \times a / 64$ grid in lateral and $a / 16$ grid in vertical dimensions. In the determination of the transmission characteristics, the finite-difference time-domain (FDTD) method is employed by using a commercial software, CrystalWave [32]. In the finite-difference time-domain (FDTD) simulations, the PC structure is arranged to have sixteen holes along the $\Gamma-K$ direction. On all the surfaces of the photonic crystal membrane, the perfectly matched boundary layers with a thickness of $2 a$ are imposed. A broadband Gaussian source is launched at the beginning of a $16 a$ long line-defect. Two sensors are placed inside the line-defect at $0.235 a$ and at $15.7 a$ away from the light source. The closest sensor to the light source is used as reference sensor and the other sensor is used as output sensor. The normalized transmission spectra are obtained by normalizing the flux data collected by the output sensor to the flux data collected by the reference sensor. All calculations are realized in three dimensions to obtain a sufficient degree of consistency with experimental observations.

The performance of the refractive index sensors are evaluated using the sensitivity parameter, $S$, which is defined as the magnitude in the shift of the cut-off wavelength divided by the change in the refractive index of the liquid

$$
S=\frac{\partial \lambda}{\partial n}
$$

\section{Results and discussions}

In an earlier study, a good agreement between CrystalWave 3D FDTD simulations and measurements was obtained for a PCW slab on $\mathrm{SiO}_{2}$ substrate with a nearly linear sensitivity of $63.69 \mathrm{~nm} / \mathrm{RIU}$ for four different cover solutions [23]. By performing 2D FDTD calculations with an effective index method for a PCW slab, it was found that substitution of all the $\mathrm{PC}$ air holes with water significantly enhances the sensing performance in comparison to substitution of the cladding layer [21]. Later, it was reported that infiltration of only the first rows of holes adjacent to the line-defect with water enhances the sensitivity greater than the infiltration of all lattice holes [18]. These reported studies indicate that selective infiltration is more beneficial than cladding and all lattice hole infiltration methods. Therefore, we follow this selective infiltration method in our analysis.

\subsection{Sensing characteristics of the PC slab waveguide}

The PC slab structure is illustrated in Fig. 1. The diameter of the lattice holes is $0.76 a$ and the slab thickness is $0.6 \mathrm{a}$. The first rows of holes adjacent to the line-defect are remarked in yellow color to emphasize that only the first rows of holes adjacent to the line-defect are infiltrated.

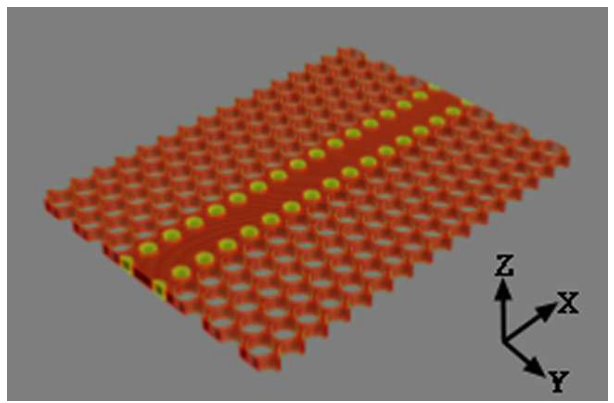

Fig. 1. The triangular lattice PC slab waveguide with only first rows of holes infiltrated.

The projected band structure for the 3D PCW structure is shown in Fig. 2. Four guided modes lie in the photonic band gap region outside the light cone. The PCW is single mode between frequencies of $0.2865(a / \lambda)$ and $0.31(a / \lambda)$ which only contain the fundamental guided mode. 


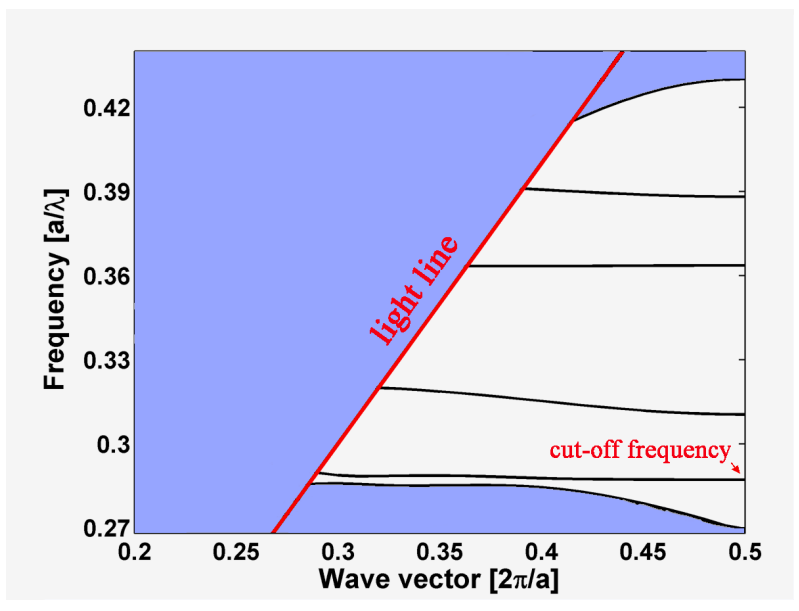

Fig. 2. The projected band structure for the PC slab waveguide with a height of $0.6 a$ and a hole diameter of $0.76 a$.

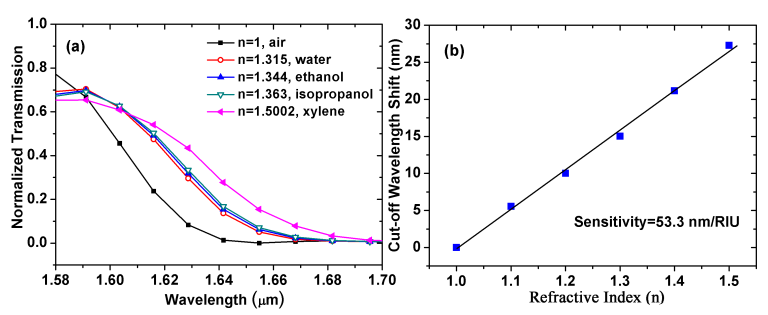

Fig. 3. (a) Transmission spectrum of the PC slab waveguide with a hole diameter of $0.76 a$ for different liquids. (b) The cut-off wavelength shift for different refractive indices.

Figure 3a shows the TE mode transmission spectra of the structure calculated by the 3D FDTD modeling for four different liquids, water $(n=1.315)$, ethanol $(n=$ $1.344)$, isopropanol $(n=1.363)$ and xylene $(n=1.5002)$. The lattice constant is set to $a=460 \mathrm{~nm}$, so that the cut-off wavelength of the guided mode corresponds to about $1.58 \mu \mathrm{m}$. The mode cut-off besides the slab modes causes a drop in the transmission spectrum for the wavelengths greater than $1.58 \mu \mathrm{m}$. As the refractive index increases,

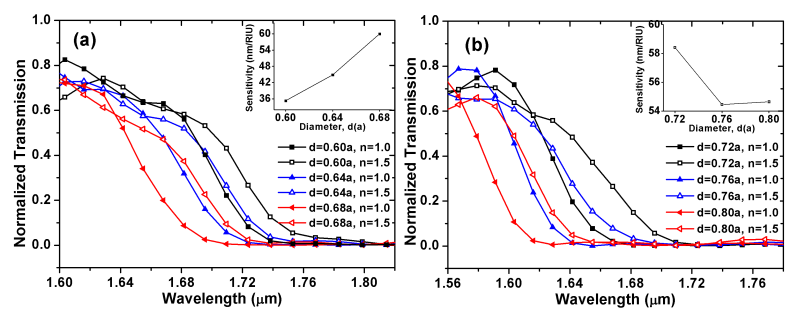

Fig. 4. (a) Transmission spectra of the PC slab waveguide for hole diameters of first rows changing from $d=0.60 a$ to $0.68 a$ and (b) from $d=0.72 a$ to $0.80 a$. The sensitivities of the PC slab waveguides as a function of hole diameter are shown in the insets. the transmission band is observed to be shifting to longer wavelengths. This is expected, since the average refractive index that the field probes increases as a result of infiltration.

Figure $4 \mathrm{~b}$ shows the cut-off wavelength shift as a function of ambient refractive index change. The cut-off wavelength, which has been read out at a normalized transmission level of 0.3 , shifts by $27.28 \mathrm{~nm}$ by the change of the local refractive index from $n=1$ to $n=1.5$. This amount of shift in this RI range corresponds to a sensitivity of $54.6 \mathrm{~nm} / \mathrm{RIU}$ (RIU denotes refractive index unit). The data points in Fig. $4 \mathrm{~b}$ follow an almost linear relation with the ambient index change leading a slope of $53.3 \mathrm{~nm} / \mathrm{RIU}$.

\subsection{Sensing characteristics for different diameters of first rows of holes}

The field distribution of the guided mode is observed to be highest at the center of the waveguide and the magnitude of field diverges as the distance along the transverse direction increases. Hence, the first rows of holes affect the dispersion characteristics more than the second and third rows [33]. The localization of field in the PCW structure can be increased by selecting the first rows of holes in a different diameter value than the background lattice holes. With this approach, the sensitivity of the sensor can be enhanced.

The transmission spectra of the PC slab waveguide are given in Fig. 4 for diameters of first rows of holes, changing from $d=0.60$ a to $0.80 a$. Since a linear characteristic response against $\mathrm{RI}$ change is observed, the transmission spectra are examined for two different RI values, $n=1$ and $n=1.5$ for further analysis. From the shift of the cut-off wavelength, the sensitivity of the device for each diameter value is calculated. The cut-off wavelength is observed at longer wavelengths as the diameter of the first rows of holes decreases. The sensitivity parameters for each diameter value are shown in the insets of Fig. 4a and $b$. As the diameter of holes in the first rows increases, the sensitivity shows an increasing behavior up to $d=0.68 a$ and a decreasing behavior with a saturation at $d=0.76 a$. The highest sensitivity, $S=60.02 \mathrm{~nm} / \mathrm{RIU}$, is obtained for the first rows of holes with $d=0.68 a$ diameter. This increase results from broadening of the transmission band for $d=0.68 a$ after infiltrating the air holes with a liquid of $n=1.5$.

The sensitivity of the PCW device, via 2D FDTD calculations, is recently reported to be four times improved by using larger holes in the first rows adjacent to the line-defect than the background holes [18]. However, in our design the maximum sensitivity is obtained for smaller holes for the first rows with a considerable enhancement in sensitivity.

\subsection{Sensing characteristics for optimized central row of holes}

Especially the waveguide region of the $\mathrm{PC}$ slab, where the field is most intense, should be utilized in order to 
reach higher sensitivity parameters. Since the field of the fundamental even mode is most intense in the center, opening sensing holes in the line-defect would benefit from the increased light-matter interaction. In this sense, an alternative infiltration procedure is followed, where only the central row of holes in the line-defect is infiltrated (Fig. 5).

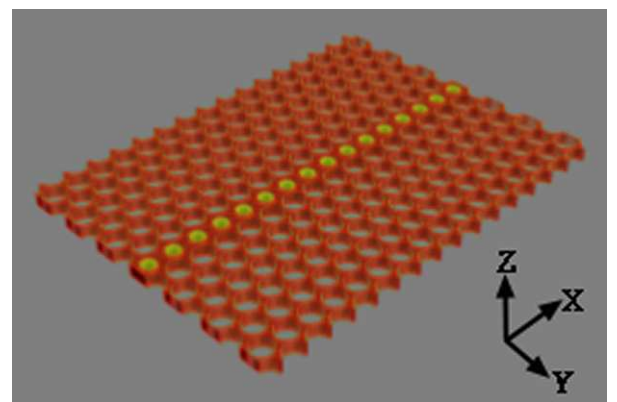

Fig. 5. The triangular lattice PC slab waveguide with only central holes infiltrated.

The projected band structure for the $3 \mathrm{D}$ PCW structure with $d=0.60 a$ diameter of central row of holes is shown in Fig. 6. Four guided modes lie in the photonic band gap region outside the light cone. The fundamental guided mode is shifted to higher frequencies due to the increase in air filling ratio with the inclusion of air holes in the center. The PCW is single mode between frequencies of $0.285(a / \lambda)$ and $0.4(a / \lambda)$ which only contains the fundamental guided mode.

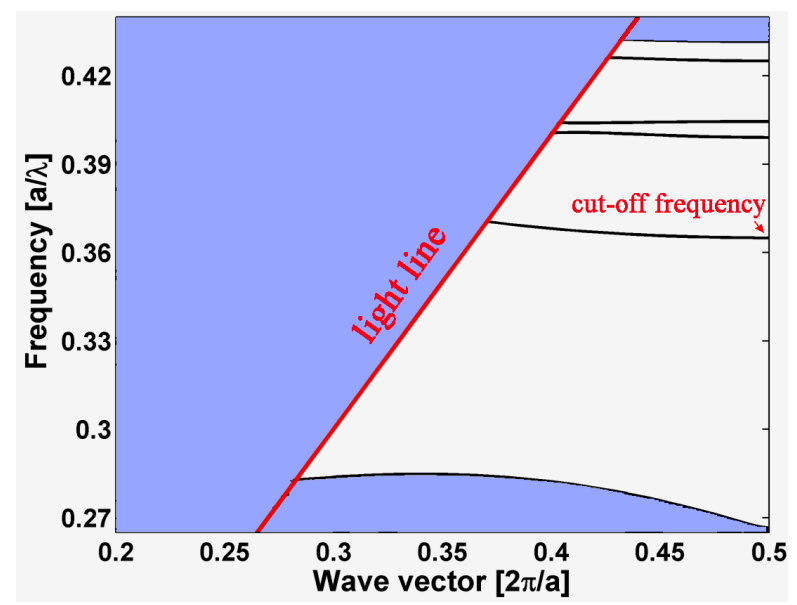

Fig. 6. The projected band structure for the PC slab waveguide with a height of $0.6 a$, background hole diameter of $0.76 a$ and central hole diameter of $0.60 a$.

The diameter of the holes in the line-defect is changed from $d=0.40 a$ to $0.60 a$. The transmission spectra related to various central hole diameters are given in Fig. 7a and $b$ with their sensitivity parameters shown in the insets. In order to obtain minimum cut-off wavelength as about $1.5 \mu \mathrm{m}$, the lattice constant is set to $560 \mathrm{~nm}$. As the diameter of central row of holes increases, the cut-off wavelength shifts to shorter wavelengths due to the increase in the low-refractive index ratio. In addition, the spectral distance between the cut-off wavelengths belonging to $n=1$ and $n=1.5$ increases with the increase of the central hole size. The sensitivity, which is calculated as $S=96.4 \mathrm{~nm} /$ RIU for $d=0.40 a$, shows a continuous increasing behavior as the central hole size increases. However, for sensing applications, not only the sensitivity parameter, but also the intensities near the cut-off wavelengths are important to gain high sensitivity. Since the sensitivity parameter is calculated at a normalized transmission level of 0.3 in this study, the increase of the central hole diameter more than $0.60 a$ would make the determination of sensitivity parameter difficult due to the decrease in signal intensity via backscattering. Therefore the central hole diameter is not increased beyond $d=0.60 a$ and the maximum sensitivity is obtained for $d=0.60 a$.

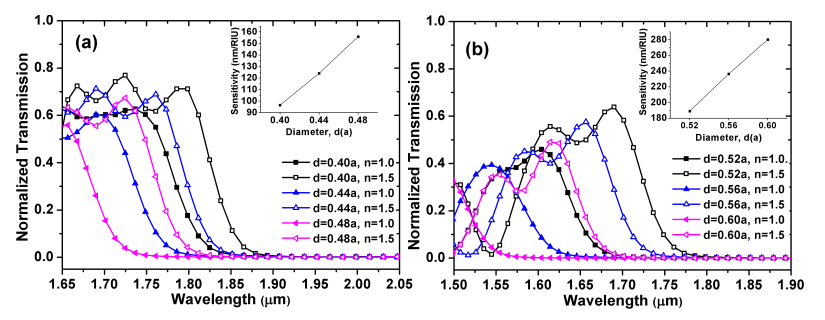

Fig. 7. Transmission spectra of the PC slab waveguide for central hole diameters changing from $d=0.40 a$ to $0.48 a$ and (b) from $d=0.52 a$ to $0.60 a$. The sensitivities of the PC slab waveguide as a function of hole diameter are shown in the insets.

Figure 8a shows the transmission spectrum of the PC slab waveguide structure with central row of holes with $d=0.60 a$ under infiltration of different liquids. As the refractive index of the liquids increase, cut-off wavelengths shift to red. This shift, as shown in Fig. 8b, shows a linear variation with the change of the refractive index of the central holes. The slope of the best line representing the data points gives $S=282.4 \mathrm{~nm} / \mathrm{RIU}$, the highest sensitivity parameter obtained among the investigated structures in this study.

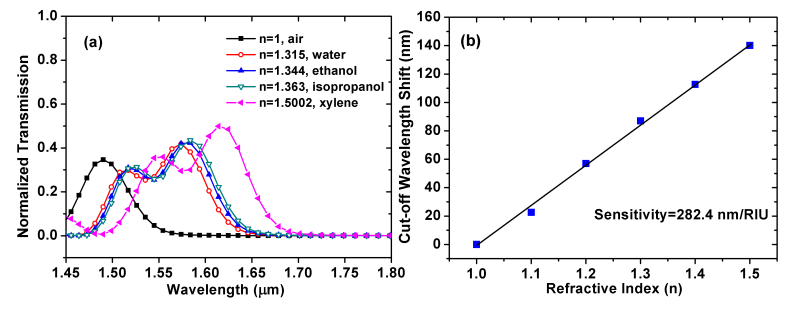

Fig. 8. Transmission spectrum of the PC slab waveguide with a central hole diameter of $0.60 a$ for different liquids. (b) The cut-off wavelength shift for different ambient refractive indices. 
The $z$-component of the magnetic field of the PC slab waveguide with $d=0.76 a$ lattice holes and its analogue with an included central row of holes with $d=0.60 a$ diameter are illustrated in Fig. 9. The even-mode field concentrates in the line-defect and in the first rows of holes for the PCW structures with air and xylene infiltrations (Fig. 9a and b). The regions where the field is positive (red color) becomes negative (blue color in Fig. 9) as xylene is infiltrated without any apparent difference in degree of field penetration. Therefore, we obtained a relatively small sensitivity for this case. When holes are included in the center, the field mainly concentrates between the central holes (Fig. 9c and d). At first glance, there seems to be no difference in the field distributions of Fig. 9c and d. However, if we closely examine Fig. 9d it can be noticed that the field penetrates more into the central holes when xylene is infiltrated. Even a slight change in the penetration depth of the field causes a large shift in the cut-off frequency, demonstrating a much higher sensitivity.

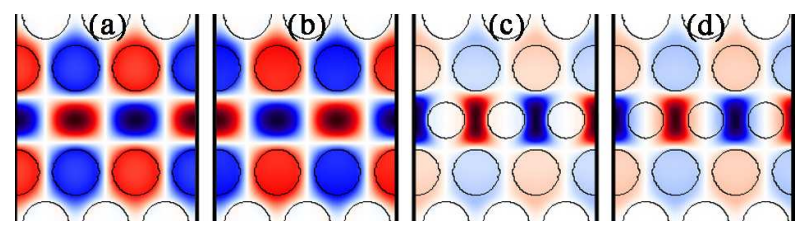

Fig. 9. $z$-component of the magnetic field in the vicinity of the liquid infiltration at the wave number of $0.5(2 \pi / a)$ for a PCW (a) without infiltration, (b) with first rows of holes infiltrated with xylene, (c) with central holes without infiltration, and (d) with central holes infiltrated with xylene.

It is noteworthy to state that the effect of the position of central holes on sensing performance is also investigated here by changing the position of the holes along and in the transverse direction to light propagation. In both cases, shifting the central row of holes is found to decrease the sensitivity. It should be noted that the immersion of the slab to the liquid is also considered in this study. However, the signal level and the sensitivity are found to be lower and the bandwidth of transmission band narrower.

The sensitivity of the PCW slab sensor with smaller holes in the line-defect is reported to be $120 \mathrm{~nm} / \mathrm{RIU}$ after immersing the structure to deionized water [20]. In this study, by infiltrating only the central row of holes, the sensitivity parameter has reached to $282.4 \mathrm{~nm} / \mathrm{RIU}$. In the light of literature, our calculations indicate that infiltrating only the holes in the line-defect has a greater impact to sensitivity than immersing the whole structure to the liquid.

\section{Conclusion}

We have designed and investigated three different photonic crystal slab waveguide structures with triangular array of holes by 3D plane-wave expansion and FDTD calculations. For all cases, we have obtained a linear response for the cut-off wavelength to changes in refractive index. The first proposed structure, a W1 PCW, leads to a sensor sensitivity of $53.3 \mathrm{~nm} / \mathrm{RIU}$ when the holes adjacent to the line-defect are infiltrated. As we optimize the radius of first rows of holes adjacent to the line-defect, the sensitivity has increased and reached to a value of $60.02 \mathrm{~nm} /$ RIU. However, a remarkable improvement in the sensitivity $(282.4 \mathrm{~nm} / \mathrm{RIU})$ is obtained by the inclusion of central row of holes and infiltration of these holes only. These results indicate that the photonic crystal slab waveguide structures with smaller infiltrated holes in the line-defect have better sensing properties. Consequently, this particular design and infiltration method can be employed in miniaturized refractive index sensing applications for better sensitivity.

\section{Acknowledgments}

We gratefully acknowledge the financial support by Scientific Research Projects of Ankara University (BAP) under grant no. 12B4343011.

\section{References}

[1] T. Endo, K. Kerman, N. Nagatani, Y. Takamura, E. Tamiya, Anal. Chem. 77, 6976 (2005).

[2] J. Homola, S.S. Yee, G. Gauglitz, Sens. Actuators B 54, 3 (1999).

[3] R.G. Heideman, R.P.H. Kooyman, J. Greve, Sens. Actuators B Chem. 10, 209 (1993).

[4] E.F. Schipper, A.M. Brugman, C. Dominguez, L.M. Lechuga, R.P.H. Kooyman, J. Greve, Sens. Actuators B Chem. 40, 147 (1997).

[5] B.J. Luff, J.S. Wilkinson, J. Piehler, U. Hollenbach, J. Ingenhoff, N. Fabricius, J. Lightwave Technol. 16, 583 (1998).

[6] R. Horvath, H.C. Pedersen, N.B. Larsen, Appl. Phys. Lett. 81, 2166 (2002).

[7] H. Li, Z. Cao, H. Lu, Q. Shen, Appl. Phys. Lett. 83, 2757 (2003).

[8] L. Rindorf, J.B. Jensen, M. Dufva, L.H. Pedersen, P.E. Hoiby, O. Bang, Opt. Express 14, 8224 (2006).

[9] S. Arnold, M. Khoshsima, I. Teraoka, S. Holler, F. Vollmer, Opt. Lett. 28, 272 (2003).

[10] T. Baehr-Jones, M. Hochberg, C. Walker, A. Scherer, Appl. Phys. Lett. 85, 3346 (2004).

[11] N.M. Hanumegowda, C.J. Stica, B.C. Patel, I.M. White, X. Fan, Appl. Phys. Lett. 87, 201107 (2005).

[12] M. Lee, P.M. Fauchet, Opt. Express 15, 4530 (2007).

[13] C. Kang, C.T. Phare, Y.A. Vlasov, S. Assefa, S.M. Weiss, Opt. Express 18, 27930 (2010).

[14] S. Mandal, D. Erickson, Opt. Express 16, 1623 (2008).

[15] M.G. Scullion, A. Di Falco, T.F. Krauss, Biosens. Bioelectron. 27, 101 (2011). 
[16] D. Dorfner, T. Zabel, T. Hurlimann, N. Hauke, L. Frandsen, U. Rant, G. Abstreiter, J. Finley, Biosens. Bioelectron. 24, 3688 (2009).

[17] S. Zlatanovic, L.W. Mirkarimi, M.M. Sigalas, M.A. Bynum, E. Chow, K.M. Robotti, G.W. Burr, S. Esener, A. Grot, Sens. Actuators B 141, 13 (2009).

[18] F. Bougriou, T. Bouchemat, M. Bouchemat, N. Paraire, in: Proc. Saudi Int. Electronics, Communications and Photonics Conf. (SIECPC) 2011.

[19] X. Wang, Q. Tan, C. Yang, N. Lua, G. Jin, Optik 123, 2113 (2012).

[20] S.C. Buswell, V.A. Wright, J.M. Buriak, V. Van, S. Evoy, Opt. Express 16, 15949 (2008).

[21] U. Erdiven, M. Karaaslan, E. Unal, F. Karadag, Acta Phys. Pol. A 122, 732 (2012).

[22] J. Topolancik, P. Bhattacharya, J. Sabarinathan, P.C. Yu, Appl. Phys. Lett. 82, 1143 (2003).

[23] N. Skivesen, A. Têtu, M. Kristensen, J. Kjems, L.H. Frandsen, P.I. Borel, Opt. Express 15, 3169 (2007).

[24] D. Erickson, T. Rockwood, T. Emery, A. Scherer, D. Psaltis, Opt. Lett. 31, 59 (2006).

[25] F. Intonti, S. Vignolini, V. Turck, M. Colocci, P. Bettotti, L. Pavesi, S.L. Schweizer, R. Wehrspohn, D. Wiersma, Appl. Phys. Lett. 89, 2111171 (2006).
[26] C.L.C. Smith, D.K.C. Wu, M.W. Lee, C. Monat, S. Tomljenovic-Hanic, C. Grillet, B.J. Eggleton, D. Freeman, Y. Ruan, S. Madden, B. Luther-Davies, H. Giessen, Y.H. Lee, Appl. Phys. Lett. 91, 121103 (2007).

[27] N. Carlsson, N. Ikeda, Y. Sugımoto, K. Asakawa, T. Takemori, Y. Katayama, N. Kawai, K. Inoue, Opt. Quantum Electron. 34, 123 (2002).

[28] L. O'Faolain, X. Yuan, D. McIntyre, S. Thoms, H. Chong, R.M. De La Rue, T.F. Krauss, Electron. Lett. 42, 1454 (2006).

[29] Y. Liu, H.W.M. Salemink, Opt. Express 20, 19912 (2012).

[30] A.V. Dyogtyev, I.A. Sukhoivanov, R.M. De La Rue, J. Appl. Phys. 107, 013108 (2010).

[31] S.G. Johnson, J.D. Joannopoulos, Opt. Express 8, 173 (2001).

[32] CrystalWave, Photon Design Ltd, developed under European Framework 5 Project "PICCO", 2003.

[33] H. Kurt, K. Üstün, L. Ayas, Opt. Express 18, 26965 (2010). 